\title{
Kommentar zu den Entwürfen des Bewirtschaftungsplans und des Maßnahmenprogramms gemäß Artikel 13 bzw. 11 WRRL für den deutschen Teil der Flussgebietseinheit Elbe
}

\author{
Ulrich Förstner
}

Online veröffentlicht: 14. Juli 2009

(C) Springer-Verlag 2009

In den Entwürfen der Bewirtschaftungspläne und Maßnahmenprogramme gemäß Artikel 13 bzw. 11 WRRL erfahren die Bestimmungen zu Sedimentaspekten der neuen Richtlinie 2008/105/EG vom 16.12.2008 eine erste Bewährungsprobe.

\section{Bewirtschaftungsplan}

Der Entwurf des Bewirtschaftungsplans und vor allem das Hintergrundpapier der Flussgebietsgemeinschaft Elbe (FGG Elbe) für den Belastungsschwerpunkt Schadstoffe hat die besonderen Merkmale der unbefriedigenden Schadstoffsituation der Elbe aufgezeigt und das Fazit gezogen, dass es sich in erheblichem Maße um ein Schwebstoff- und Sedimentproblem handelt. Auf der Grundlage einer Auftragsstudie der FGG Elbe und der Hamburg Port Authority (HPA) über die Risiken durch feststoffgebundene Schadstoffe wurden erste Reduktionsforderungen abgeleitet, die in den einzelnen Teileinzugsgebieten im deutschen Teil der Flussgebietseinheit Elbe umgesetzt werden sollen.

\section{Maßnahmenprogramm}

Während die sedimentrelevanten Konzepte im Bewirtschaftungsplan der FGG Elbe überwiegend dem Stand der wissenschaftlichen Diskussionen in der EU entsprechen,

U. Förstner $(\bowtie)$

Technische Universität Hamburg-Harburg, Institut für Umwelttechnik und Energiewirtschaft, Eissendorferstr. 40, 21071 Hamburg, Deutschland E-Mail: u.foerstner@tu-harburg.de spiegeln die entsprechenden Maßnahmenprogramme noch immer die Einstellung weiter Kreise der europäischen Wasserpolitik wieder, die der Sedimentthematik keine Bedeutung bei der Umsetzung der WRRL beimessen wollen. So enthalten weder der Umweltbericht zum Entwurf des Maßnahmenprogramms gemäß Art. 11 der WRRL für die FGG Elbe noch der standardisierte Maßnahmenkatalog der Bund/ Länder-Arbeitsgemeinschaft Wasser (LAWA) mit seinen 99 umsetzungsbezogenen und acht konzeptionellen Maßnahmen keine Hinweise auf die praktische Vorgehensweise bei Sedimentaltlasten (,historisch kontaminierte Sedimente“). Auf die Maßnahmenvorschläge der Auftragsstudie ist der Entwurf des Bewirtschaftungsplans bislang nicht eingegangen.

\section{3 ,1:1-Umsetzung"6 oder ,Geist der WRRL“?}

Die Defizite im Bereich der Maßnahmenprogramme für Sedimente zeigen, dass sich die Politik und Administration in besonders kritischen Situationen nicht auf eine 1:1-Umsetzung der Wasserrahmenrichtlinie zurückziehen darf. Beispielsweise lässt sich die weitere Verzögerung der längst überfälligen Sanierung der Dioxinaltlast im Spittelwasser, für die bereits 1993 eine Machbarkeitsstudie vorgelegt wurde, nicht durch die Unterscheidung von ,grundlegenden“ und ,ergänzenden“ Maßnahmen begründen; jedes künftige Hochwasserereignis kann für die unterliegenden Überflutungsflächen unübersehbare Nachteile mit sich bringen. Besonders problematisch sind die sedimentbürtigen Schadstoffeinträge für die marinen Ökosysteme; ausgerechnet für die elbetypischen Dioxine gibt es keinen Richt- oder Zielwert, mit dem die Umlagerung bzw. Verklappung von Sedimenten im Tidebereich und in der Nordsee überwacht werden könnte. 